\title{
MINIMAL PRIME IDEALS AND COMPACTIFICATIONS
}

\author{
J. H. RUBINSTEIN
}

(Received 30 June 1969; revised 4 February 1970)

Communicated by B. Mond

\section{Introduction}

Following on from the work of T. P. Speed [1], we will deal with some obvious conjectures, using particularly results 3.3 and 8.5 of [1].

Our notation will be the same as [1]. All lattices considered will be distributive with zero. The main question is whether a disjunctive lattice is uniquely characterized by its minimal prime ideal space. By 8.1 of [1], an arbitrary lattice $L$ is isomorphic to the lattice $\mu_{\mathscr{L}}$ under the mapping $x \rightarrow \mathscr{M}(x)$ iff $L$ is disjunctive; and by 4.1 of [1], $\operatorname{Min} p(\mathscr{L}) \cong \operatorname{Min} p\left(\mu_{\mathscr{L}}\right)$. So when considering how much minimal prime ideal spaces reflect the properties of lattices we need only consider disjunctive lattices. The answer to this question turns out to be negative, and under quite general conditions, there appear to be many disjunctive lattices with the same minimal prime ideal space.

In section 3 is given an example to this effect which also shows that the conjecture that "The minimal prime ideal space of a disjunctive lattice being locally compact implies that the lattice is generalised Boolean" is false.

Next the main result of [2] is treated in a more simple manner in section 4 (for an earlier and better proof see [4] pp. 24-28). Finally the connection between prime ideal spaces and compactifications is used in sections $5-7$ to try to find which disjunctive lattices give the same minimal prime ideal space. Only a small amount of progress is made on this problem.

\section{2. $\zeta$-spaces}

Definition. A Hausdorff space $(X, \mathscr{I})$ is called a 0-dimensional space if there is a basis of open closed subsets of $X$ for the topology $\mathscr{I}$. This definition is from Bourbaki.

Definition. A topological space $(X, \mathscr{I})$ is called a $\zeta$-space when the following holds.

(i) $(X, \mathscr{I})$ is a 0 -dimensional space

(ii) There is a basis $B$ of open closed subsets of $X$ such that the topology $\tilde{\mathscr{I}}$ 
generated by the family $\left\{b^{\mathrm{c}}: b \in B\right\}$ (denoted $\left.B^{\mathrm{c}}\right)$ is such that $(X, \tilde{\mathscr{I}})$ is a compact space.

Definition. A basis for a $\zeta$-space $(X, \mathscr{I})$ satisfying the condition (ii) above is called a $\zeta$-basis.

Clearly there is no loss of generality in considering all bases as closed under finite unions and intersections and we assume so from now on.

Propositions 3.1, 3.3 and 8.5 of [1] say the following:

Minimal prime ideal spaces are always $\zeta$-spaces, with one $\zeta$-basis being $\mu_{\mathscr{L}}$ (which is $i$ somorphic as a lattice to the original lattice). If a $\zeta$-basis is considered as a lattice, then its minimal prime ideal space is homeomorphic to the $\zeta$-space.

We shall use the following well known and easy result:

A 0 -dimensional space $(X, \mathscr{I})$ is locally compact iff there is a basis for $\mathscr{I}$ consisting of the family of all open closed compact sets (see for example Lemma 2.4 of [2]), and this basis is contained in any other basis of open closed sets for $\mathscr{I}$ (Lemma 2.10 of [2]).

LEMMA. A 0-dimensional space $(X, \mathscr{I})$ which is locally compact has a $\zeta$-basis for $\mathscr{I}$ consisting of the family of all open closed compact sets. Hence $(X, \mathscr{I})$ is a $\zeta$-space.

Proof. Let $B$ be the family of all open closed compact subsets of $X$. Suppose $\tilde{\mathscr{I}}$, the topology generated by $\left\{b^{\mathrm{c}}: b \in B\right\}$, is not compact. Then there is a family of elements of $B$ with the FIP, say $\left\{b_{\alpha}: \alpha \in A\right\}$ such that $\bigcap_{\alpha \in \mathrm{A}} b_{\alpha}=\square$. This follows since $B$ generates all the closed sets in $\tilde{\mathscr{I}}$ by arbitrary intersections. But this contradicts the fact that each $b_{\alpha}$ is a closed compact set.

COROLlaRY. If a 0 -dimensional space $(X, \mathscr{I})$ is compact then it is a $\zeta$-space and the only $\zeta$-basis for $\mathscr{I}$ is the family of all open closed subsets of $X$.

Proof. See the result stated just before the lemma.

REMARK. A $\zeta$-space is compact iff the family of all open closed sets is a $\zeta$-basis. This $\zeta$-basis when considered as a lattice is Boolean.

REMARK. An example of a non locally compact $\zeta$-space is the following. Let $S$ be an uncountable set with the discrete topology. Let $X=S \cup\{v\}$ where a set containing $v$ is said to be open iff its complement in $X$ is countable. Then a $\zeta$-basis for $X$ is the singletons of $S$ plus all open (closed) sets containing $v$.

\section{Example: Non-isomorphic Disjunctive Lattices with the Same Minimal Prime Ideal Space}

To consider the questions outlined at the beginning, let $(X, \mathscr{I})$ be an arbitrary Iocally compact 0 -dimensional space. Denote by $B$ the family of all open closed compact subsets of $X$. We will assume also that $(X, \mathscr{I})$ is not compact. 
Proposition. If $d$ is an arbitrary open closed subset of $X$ which is not a compact subset of $X$ then the basis for $\mathscr{I}$ generated by $B \cup\{d\}$ is a $\zeta$-basis for $(X, \mathscr{I})$.

Proof. Denote by $D$ the basis generated by $B \cup\{d\}$. Then an arbitrary element $y \in D$ is of the form $y=b, b \in B$ or $y=b \cup d$ where $b \in B$. This follows since for example $d \cap b$ is an open closed compact set for $b \in B$ and so $d \cap b$ belongs to $B$.

Denote by $(X, \mathscr{V})$ the topological space on $X$ generated by $\left\{y^{\mathrm{c}}: y \in D\right\}$. Suppose that $(X, \mathscr{V})$ is not compact. Then since the closed sets of $\mathscr{V}$ are intersections of sets- of the form $b, b \cup d$ where $b \in B$ it follows that

$$
\bigcap_{\delta} b_{\delta} \cap \bigcap_{\gamma}\left(b_{\gamma} \cup d\right)=\square
$$

where $\left\{b_{\delta}, b_{\gamma} \cup d\right\}_{\delta \varepsilon \mathrm{A}},{ }_{\gamma \in \mathrm{C}}$ has the FIP. So clearly

$$
\begin{aligned}
& \bigcap_{\delta} b_{\delta} \cap d=\square, \\
& \bigcap_{\delta} b_{\delta} \bigcap_{\gamma} b_{\gamma}=\square .
\end{aligned}
$$

By the lemma we have that $B$ is a $\zeta$-basis and so the family $\left\{b_{\delta}, b_{\gamma}\right\}_{\delta_{\varepsilon A} \text {, } \gamma_{\varepsilon C} \mathrm{C}}$ cannot have the FIP. Without loss of generality we may assume $b_{\delta_{1}} \cap b_{\gamma_{1}}=\square$ for some $\delta_{1}$ and $\gamma_{1}$. So using the FIP of the original family it follows that

$$
b_{\delta_{1}} \cap\left(b_{\gamma_{1}} \cup d\right)=b_{\delta_{1}} \cap d \neq \square \text {. }
$$

Then, since

$$
b_{\mathrm{\delta}} \cap b_{\delta_{1}} \cap\left(b_{\gamma_{1}} \cup d\right)=b_{\delta_{1}} \cap b_{\delta} \cap d \neq \square,
$$

we must have $b_{\delta} \cap d \neq \square$ for any $\delta$. Hence $\left\{b_{\delta} \cap d\right\}_{\delta \varepsilon A}$ is a FIP family and is, clearly contained in $B$. Then (1) contradicts $B$ a $\zeta$-basis.

In exactly the same way it can be shown that any finite number of noncompact open closed sets can be added to $B$ and the result is still a $\zeta$-base.

We quickly show that $B$ and $D$ are not lattice isomorphic. $B$ is easily seen to be a generalised Boolean lattice. Suppose $D$ is generalised Boolean. Then in particular the principal ideal $(d)_{D}$ generated by $d$ in $D$ must be a Boolean lattice. As $d$ is open closed and non compact, clearly there exists $b \in B$ with $0 \neq b \subset d$. So $d \cap b^{c}$, which is the complement in $(d)_{D}$ of $b \in(d)_{D}$, must belong to $(d)_{D}$ and hence to $D$, since $(d)_{D}$ is Boolean. Now $d \cap b^{c} \in D$ means either $d \cap b^{c} \in B$ or $d \cap b^{c}$ contains $d$. The latter is obviously impossible, so $d \cap b^{c} \in B$. Thus $d=(d \cap b) \cup\left(d \cap b^{c}\right)$ and both of the summands are compact and in $B$. Therefore $d \in B$, a contradiction.

By Lemma 3.3 of [5], any basis of open closed sets in a 0-dimensional space is a disjunctive lattice. So $B$ and $D$ are both disjunctive lattices. By proposition 8.5 of [1], it follows that

$$
\operatorname{Min} p(B) \cong(X, \mathscr{I}) \cong \operatorname{Min} p(D) .
$$

So putting all the results together gives an example of two non lattice-isomorphic 
disjunctive lattices with the same minimal prime ideal space. Also, since $D$ is not a generalised Boolean lattice it follows that the condition that the minimal prime ideal space of a disjunctive lattice is locally compact does not imply that the lattice is generalised Boolean.

REMARK. (This was kindly suggested by the referee). There can be no example of a minimal prime ideal space of two non lattice-isomorphic disjunctive lattices with one or both of the lattice finite.

The reason is that any finite distributive disjunctive lattice with $\mathbf{0}$ is Boolean. Firstly any such lattice $\mathscr{L}$ clearly has a finite and thus compact minimal prime ideal space. Hence by the Corollary in section 2, the only $\zeta$-basis for $\operatorname{Min} p(\mathscr{L})$ is a Boolean lattice. So $\mathscr{L} \simeq \mu_{\mathscr{L}}$ are both Boolean, for as already remarked in section $2, \mu_{\mathscr{L}}$ is a $\zeta$-basis for $\operatorname{Min} p(\mathscr{L})$.

So any such lattice has $2^{n}$ elements, where $n$ is the number of atoms (= number of minimal prime ideals) of the lattice. Thus, if one of the two lattices is finite, so must be the minimal prime ideal space and the other lattice. Hence both lattices are finite Boolean with the same number of atoms, thus the same number of elements; so they are isomorphic (Halmos, Lectures on Boolean Algebras, p 72 (exercise)). In this case the construction of a new $\zeta$-basis $D$ from the old one $B$ fails because the (finite) topological space is compact and so there are no noncompact open closed sets to be added to $B$.

\section{0-Dimensional compactifications of 0 -dimensional spaces}

We will now turn to the question of compactifications of a 0-dimensional space and the relation this has to minimal prime and prime ideals. The most natural way of getting such compactifications as considered in [1] and [2] is as follows:

Let $(X, \mathscr{I})$ be a 0 -dimensional space. We will denote points of $X$ by Greek letters and subsets of $X$ by small Latin letters. Let $D$ be a Boolean lattice of openclosed subsets of $X$ such that $D$ is a basis for the topology $\mathscr{I}$. Let Spec $D$ be the space of prime ideals of $D$ with the hull-kernel topology.

Lemma. The mapping $\tau$ from $X$ to Spec. $D$ given $b y$

$$
\tau(\xi)=\{d \in D: \xi \notin d\}
$$

is a homeomorphism from $X$ onto a dense subspace of $\operatorname{Spec} D$.

Proof. The fact that $\tau X$ is dense follows since kernel $(\tau X)=\cap \tau X=\square$. See Lemma 4.3 of [5] for the full proof. We can now identify $X$ with $\tau X$ and so can consider $X$ to be embedded in Spec $D$.

Lemma. Since $D$ is a Boolean lattice, Spec $D$ is a compact Hausdorff space. Also there is a lattice isomorphism between $G$, the family of all open closed subsets of Spec $D$, and $D$ itself. 
Proof. This is just the well known result of [6] for the case of a Boolean lattice. See also Th. 7.4 of [5] and Page 762 of [2]. In fact

$$
G=\{\mathscr{P}(d): d \in D\} \text { where } \mathscr{P}(d)=\{P \in \operatorname{Spec} D: d \notin P\} .
$$

Then the lattice isomorphism between $G$ and $D$ is given by $\mathscr{P}(d) \rightarrow d$. When $X$ is identified with $\tau X$, the useful relationship

$$
\mathscr{P}(d) \cap X=d \text { holds. }
$$

We will show later that a similar result holds for $\Gamma$ any 0-dimensional compactification of $(X, \mathscr{I})$. The main result of [2] (Th. 3.6 p. 762) (see also [4] pp. 24-28) is the following, which we divide into two parts for ease of proof.

THEOREM 1. Let $\mathscr{D}$ be the family of all Boolean lattices of open closed sets which form a basis for $(X, \mathscr{I})$ and let $\operatorname{Spec} \mathscr{D}=\{\operatorname{Spec} D: D \in \mathscr{D}\}$. Order $\mathscr{D}$ by inclusion and order $\operatorname{Spec} \mathscr{D}$ by $\operatorname{Spec} D_{1} \leqq \operatorname{Spec} D_{2}$ iff there is a continuous map $f$ from $\operatorname{Spec} D_{2}$ onto Spec $D_{1}$ such that $X$ is fixed relative to $f$. Then $\mathscr{D}$ and $\operatorname{Spec} \mathscr{D}$ are order isomorphic.

THEOREM 2. The family of all 0-dimensional compactifications of $(X, \mathscr{I})$ coincides with Spec $\mathscr{D}$, where homeomorphic compactifications relative to $X$ are not distinguished (i.e. $X$ is fixed under each such homeomorphism).

Proof of Theorem 1. That $\mathscr{D} \rightarrow \operatorname{Spec} \mathscr{D}$ is a bijection is clear from the correspondence between $G_{\mathrm{Spec} \mathrm{D}}$ and $D$.

To show $\mathscr{D} \rightarrow$ Spec $\mathscr{D}$ is an order isomorphism clearly we need only show $D_{1} \subset D_{2}$ iff Spec $D_{1} \leqq \operatorname{Spec} D_{2}$. Suppose $D_{1} \subseteq D_{2}$. Then for any $P_{2} \in \operatorname{Spec} D_{2}$, $P_{2} \cap D_{1} \in \operatorname{Spec} D_{1}$. So $f: P_{2} \rightarrow P_{2} \cap D_{1}$ is a well defined mapping from $\operatorname{Spec} D_{2}$ to $\operatorname{Spec} D_{1}$.

To show: $f$ fixes $X$, is onto, and is continuous. Let $\xi \in X$ be arbitrary. We must show $f\left(\tau_{2}(\xi)\right)=\tau_{1}(\xi)$ where $\tau_{i}: \xi \rightarrow\left\{d_{i}: \xi \notin d_{i}\right.$ and $\left.d_{i} \in D_{i}\right\} i=1,2$.

But then $f\left(\tau_{2}(\xi)\right)=\left\{d_{2}: \xi \notin d_{2}, d_{2} \in D_{2}\right\} \cap D_{1}=\left\{d_{1}: \xi \notin d_{1}, d_{1} \in D_{1}\right\}$ since $D_{1} \subseteq D_{2}$. Hence $f$ fixes $X$. Suppose $P_{1}$ is any element of Spec $D_{1}$. Take $P_{2}$ as the prime ideal of $D_{2}$ generated by $P_{1}$ (well defined since $D_{2}$ is Boolean; ideals are prime iff maximal).

Then $f\left(P_{2}\right)=P_{2} \cap D_{1} \supseteq P_{1}$.

Also since $D_{1}$ is Boolean, $P_{1}$ is a maximal ideal. So if the above inclusion is proper then $P_{2} \cap D_{1}=D_{1}$ and so $X \in P_{2}$, which contradicts $P_{2}$ a proper ideal. So $f\left(P_{2}\right)=P_{1}$ and $f$ is onto.

Finally $f$ is continuous follows since if $\mathscr{P}_{1}\left(d_{1}\right)$ is a basic open (closed) subset of $\operatorname{Spec} D_{1}$ then $f^{-1}\left(\mathscr{P}_{1}\left(d_{1}\right)\right)=\left\{P_{2}: P_{2} \in \operatorname{Spec} D_{2}\right.$ and $\left.d_{1} \notin P_{2}\right\}=\mathscr{P}_{2}\left(d_{1}\right)$ which is also open (closed) in Spec $D_{2}$.

Conversely suppose $f: \operatorname{Spec} D_{2} \rightarrow \operatorname{Spec} D_{1}$ is a continuous onto mapping which fixes $X$. Then for any $d_{1} \in D_{1}$, there exists $d_{2} \in D_{2}$ such that $f^{-1}\left(\mathscr{P}_{1}\left(d_{1}\right)\right)=$ 
$\mathscr{P}_{2}\left(d_{2}\right)$. But $\mathscr{P}_{1}\left(d_{1}\right) \cap X=d_{1}$. This means $f^{-1}\left(\mathscr{P}_{1}\left(d_{1}\right)\right) \cap X=d_{1}$ since $X$ is fixed. So $\mathscr{P}_{2}\left(d_{2}\right) \cap X=d_{1}=d_{2}$ and this is just $D_{1} \subseteq D_{2}$.

Proof of TheOREM 2. Let $\Gamma$ be a 0 -dimensional compactification of $(X, \mathscr{I})$. Firstly we will show there is a bijection between $\mathscr{G}$, the family of all open-closed subsets of $\Gamma$, and $D$, for some $D \in \mathscr{D}$.

Define a mapping $f$ by $f: G \rightarrow G \cap X$ for all $G \in \mathscr{G}$. Then $\{f(G): G \in \mathscr{G}\} \in \mathscr{D}$ because $\mathscr{I}$ on $X$ is induced by the topology on $\Gamma$, and so we can take this family as $\mathscr{D}$.

We need only show $f$ is $1-1$. Suppose $G_{1} \cap X=G_{2} \cap X$. Then $\left(G_{1}-G_{2}\right) \cap$ $X=\square$. But $X$ is dense in $\Gamma$ and so $G_{1}-G_{2}=\square$. So $G_{2}-G_{1}=\square$ also and finally $G_{1}=G_{2}$.

Taking $D=\{f(G): G \in \mathscr{G}\}$ we will show that $\Gamma$ and Spec $D$ are homeomorphic with respect to a function which fixes $X$. Clearly $D$ and $\mathscr{G}$ are lattice isomorphic; consequently $\operatorname{Spec} D \cong \operatorname{Spec} \mathscr{G}$. Also $\Gamma$ is itself a 0 -dimensional space with $\mathscr{G}$ as the Boolean lattice of all open closed sets, which clearly forms a basis. So we can consider $\operatorname{Spec} \mathscr{G}$ as a compactification of $\Gamma$. But then under the usual embedding of $\Gamma$ in Spec $\mathscr{G}$, it follows that $\Gamma$ is a compact subset of a Hausdorff space and so is closed. But $\Gamma$ is also dense in Spec $\mathscr{G}$ and so $\Gamma$ and Spec $\mathscr{G}$ coincide under the embedding.

Hence $\Gamma$ and Spec $\mathscr{G}$ and so $\Gamma$ and Spec $D$ are homeomorphic. It is easily verified that $X$ is fixed under this homeomorphism.

REMARK. This shows that if $\mathscr{G}$ is a Boolean lattice of open closed subsets which forms a basis for some compact 0-dimensional space $\Gamma$, then $\Gamma$ and $\operatorname{Spec} \mathscr{G}$ are homeomorphic.

\section{5. $\zeta$-Spaces, minimal Boolean extensions, and compactifications}

Now to return to the problem of $\zeta$-spaces i.e. minimal prime ideal spaces, and the connection between them and compactifications.

Let $(X, \mathscr{I})$ be a $\zeta$-space with $B$ a $\zeta$-basis of open closed subsets of $X$. Then we wish to consider the relationship between B and the minimal Boolean lattice $D$ of open-closed sets containing $B$. If we assume as usual that $(X, \mathscr{I})$ is not compact then it follows that $B$ is not Boolean. In the example of a locally compact $\zeta$-space as discussed previously, the complement of any member of the smallest $\zeta$-basis of compact open closed subsets can be adjoined to give a larger $\zeta$-basis. Clearly the two $\zeta$-bases are non isomorphic (the larger has a non-trivial centre and a unit) while they both give rise to the same minimal Boolean lattice, that of all open closed compact sets and their complements.

A partial description of the family of $\zeta$-bases which generate the same minimal Boolean fatcice is given in section 7 . We need the following result which is contained implicitly in [3]. 
THEOREM 3. Let $D$ be a Boolean lattice which is the minimal Boolean extension of some lattice $B$ which is distributive and contains 0 and 1 . Consider $D$ as embedded in Spec $D$. If $\Gamma$ is the underlying set for Spec $D$ then $\Gamma$ can be retopologized by taking $B$, embedded in $\Gamma$, as a basis, which gives $\operatorname{Spec} B$ as the new topological space.

Note. The process can be reversed to give Spec $D$ as a new topology on Spec $B$ (see [3], Th. 2.1].

Proof. Firstly we will show there is a bijection between $\operatorname{Spec} D$ and $\operatorname{Spec} B$.

Define a mapping by $f: P \rightarrow P \cap B$ for all $P \in \operatorname{Spec} D$. Then $f$ is a well defined map from Spec $D$ to Spec $B$, in the case that $B$ has a 1 . For assume that $B$ has a 1 . Then supposing that $P \cap B=B$ holds gives the conclusion that $P$ is not a proper ideal of $D$, a contradiction.

Suppose $P_{1} \cap B=P_{2} \cap B$. Since $D$ is Boolean, for any $b \in B$ exactly one of $b \in P$ or $b^{c} \in P$ holds for any $P \in \operatorname{Spec} D$.

But then $b \in P_{1}$ iff $b \in P_{2} \quad b^{c} \in P_{1}$ iff $b^{c} \in P_{2}$ must follow.

Finally $b_{1} \cup b^{c} \in P_{1}$, iff $b_{1} \in P_{1}$ and $b \notin P_{1}$ iff $b_{1} \cup b^{c} \in P_{2}$

$$
b_{1} \cap b^{c} \in P_{1} \text {, iff } b_{1} \in P_{1} \text { or } b \notin P_{1} \text { iff } b_{1} \cap b^{c} \in P_{2} \text {. }
$$

So $P_{1}=P_{2}$ as $D=\left\{b, b^{c}, b_{1} \cup b^{c}, b_{1} \cap b^{c}\right\}$.

To show $f$ is onto, if $Q$ is a prime ideal of $B$ then define $P \in \operatorname{Spec} D$ by

$$
\begin{array}{lll}
b \in P \text { iff } b \in Q & b_{1} \cup b^{c} \in P \text { iff } b_{1} \in Q \text { and } b \notin Q \\
b^{c} \in P \text { iff } b \notin Q & b_{1} \cap b^{c} \in P \text { iff } b_{1} \in Q \text { or } b \notin Q .
\end{array}
$$

Then clearly $P \cap B=Q$ and so $f$ is a bijection.

Finally $B$ is a basis for Spec $B$ on $\Gamma$ since by the embedding of $D$ in Spec $D$, $b \in B \subseteq D$ corresponds to $\mathscr{P}(b) \in \operatorname{Spec} D=\Gamma$ under the same embedding. If we now use the mapping $f$ to identify $\Gamma$ with Spec $B$, the subset corresponding to $b$ becomes $\{P \cap B: P \in \mathscr{P}(b)\}=\{Q: Q \in \operatorname{Spec} B$ and $b \notin Q\}=\mathscr{P}(b)$ taken in Spec $B$ (denote by $\mathscr{P}_{\mathbf{B}}(b)$ ).

So clearly $B$ is a basis for $\operatorname{Spec} B$ on $\Gamma$.

Remarks. The assumption that $B$ has a unit does not imply any loss in generality for the following reasons.

Suppose $B$ is a distributive lattice without a unit (but with a zero as throughout this paper).

Adjoin a unit to $B$ to give a new lattice $B^{\prime}$ i.e. $B^{\prime}=B \cup\{1\}$. The only difference between the prime ideal spaces of $B$ and $B^{\prime}$ is that $B^{\prime}$ has a single maximum prime ideal added.

In fact Spec $B^{\prime}$ is just $\operatorname{Spec} B$ with a single point added to each closed subset, which obviously gives a compactification of $\operatorname{Spec} B$. Also since we are interested primarily in the minimal prime ideal space of $B$ which is unaltered, there is no restriction of generality. (Min $p(B)$ identical with $\operatorname{Min} p\left(B^{\prime}\right)$ corresponds to the 
fact that $B$ a $\zeta$-basis for $(X, \mathscr{I})$ such that $X \notin B$ then $B \cup\{X\}$ is also a $\zeta$-basis for $(X, \mathscr{I}))$. We can get a similar compactification in a different way as follows.

Take $B$ a distributive lattice without a unit. As in [3] form the topology on Spec $B$ given by taking $\{\mathscr{P}(b), h(b)\}=\left\{\mathscr{P}(b), \mathscr{P}(b)^{c}\right\}$ as a basis for the topology

Then it can easily be shown that this new topology on the space $\Gamma$ of Spec $B$ gives Spec $C$ where $C$ is the minimal generalised Boolean lattice containing $B$. This result follows exactly as the result in [3].

We can then get $\operatorname{Spec} D$, where $D$ is the minimal Boolean extension of either $B$ or $C$ by taking the Alexandroff one point compactification of Spec $C$. This method is applicable since Spec $C$ has a basis of compact open closed subsets for its topology. The Spec $D$ topology relativized to $\Gamma$ provides the new topology Spec $C$ of the above result.

\section{Application to 0-dimensional spaces}

Theorem 3 can be applied to the case of a 0 -dimensional space $(X, \mathscr{I})$ as follows.

Let $D$ be a Boolean lattice of open closed subsets which is a basis for $\mathscr{I}$ and has a sublattice $B$ such that $\square, X \in B$, and $D$ is the minimal Boolean extension of $B$.

Then as before Spec $D$ is a compactification of $(X, \mathscr{I})$. Let $\Gamma$ be the underlying set for $\operatorname{Spec} D$. The topology of $\operatorname{Spec} B$ on $\Gamma$ is then generated by $\{\mathscr{P}(b): b \in B\}$ which is the family of open closed subsets of Spec $D$ which correspond to $B$.

(When $(X, \mathscr{I})$ is compact it follows that $(X, \mathscr{I})$ and Spec $D$ coincide by the remark following the proof of theorem 2 , and so we are back in the case of theorem 3.)

Now we wish to consider which points of $\Gamma$ correspond to the minimal prime ideals of $B$. Let $Y$ be the subset of $\Gamma$ consisting of these points. Under the embedding of $X$ in $\Gamma, \xi \in X$ corresponds to $\{d: d \in D$ and $\xi \notin d\}$ in Spec $D$. Thus it corresponds to $\{b: b \in B$ and $\xi \notin b\}$ in Spec $B$. Denote this prime ideal by $M_{\xi}$.

THEOREM 4. The following are equivalent

1. $X \subseteq Y=\{$ points of $\Gamma$ corresponding to minimal prime ideals $\}$

2. $b^{c}$ is a union of elements of $B$, for all $b \in B$.

3. $B$ is a basis for $\mathscr{I}$ on $X$.

4. Spec $B$ induces $\mathscr{I}$ on $X$.

Proof. $1 \Leftrightarrow 2$. A prime ideal $P$ is minimal iff for any $b \in P$ there exists $b^{\prime} \notin P$ such that $b \cap b^{\prime}=\square$ (see lemma 3.1, [5]). So the $M_{\xi}$ are minimal for every $\xi \in X$ iff for any $b \in B$ and $\xi \notin b$, there exists a $b^{\prime} \in B$ such that $\xi \in b^{\prime}$ and $b \cap b^{\prime}=\square$ But this is just the condition that $b^{c}$ is a union of elements of $B$.

$3 \Leftrightarrow 4$. Since $\mathscr{P}(b) \cap X=b$ as noted previously, it follows that $\operatorname{Spec} B$ on $\Gamma$ 
induces the topology on $X$ given by taking $B$ alone as basis. Thus $B$ is a basis for the topology $\mathscr{I}$ on $X$ iff $\operatorname{Spec} B$ induces the topology $\mathscr{I}$ on $X$.

$2 \Rightarrow 3$. Assuming 2, it follows that the topology generated by $B$ on $X$ equals the topology generated by $D$ on $X$, as $D$ is the minimal Boolean extension of $B$. Since we have assumed $D$ is a basis for $\mathscr{I}$, the result follows.

$3 \Rightarrow 2 . b^{c}$ is open closed in the topology $\mathscr{I}$, because $b$ is.

Corollary. Suppose B is a basis for the topology $I$ on $X$. Then $B$ is a $\zeta$-basis and $X$ is a $\zeta$-space iff $Y$ and $X$ coincide.

Finally if $(X, \mathscr{I})$ is compact then since $B$ has a 1 it can be easily seen that Spec $B$ and Min $p B=Y$ coincide iff $B$ and $D$ coincide. This is just the special case of $B$ Boolean of the well known result that Spec $B$ is Hausdorff exactly when $B$ is generalised Boolean.

\section{Equivalent $\zeta$-bases}

Our final result is a partial characterization of the family of all $\zeta$-bases which generate the same Boolean lattice.

Let $B$ be a $\zeta$-basis in the $\zeta$-space $(X, \mathscr{I})$. We will assume as before that $B$ has a unit i.e. $X \in B$. We can identify $b \in B$ with $\mathscr{M}(b)=\{M: M \in \operatorname{Min} p(B)$ and $b \notin M\}$.

When considering $B$ as a disjunctive lattice we will write the elements of $B$ as $b \in B$. However in $(X, \mathscr{I})$ we will write them as $\mathscr{M}(b) \in B$ instead . Also denote $\mathscr{M}\left(b^{c}\right)$, i.e. the corresponding element for $b^{c}$, as $h(b)=\{M: M \in \operatorname{Min} p(B)$ and $b \in M\} . b^{c} \notin B$ corresponds exactly to the case when $b \notin$ centre of $B$, denoted $Z(B)$.

We wish to find a condition for when such $b^{c}$ can be added to $B$ and the result is still a $\zeta$-basis.

THEOREM 5. Under the above assumptions, if $b \notin Z(B)$ then $b^{c}$ cannot be added to $B$ iff there exists a prime ideal $P \in \operatorname{Spec} B$ such that $b \in P$ and for any minimal prime ideal $M \subseteq P$ implies that $b \notin M$.

Proof. $b^{c}$ not being able to be added to $B$ is equivalent to $X$ being not compact in the dual topology $\mathscr{I}$ generated by $B^{c} \cup\{b\}$. This means that there exists $\left\{b_{\alpha}\right\}_{\alpha \in A} \subset B$ such that

$$
\bigcap_{a \in A} \mathscr{M}\left(b_{\alpha}\right) \cap h(b)=\square
$$

where $\left\{\mathscr{M}\left(b_{\alpha}\right), h(b)\right\}$ is a FIP family. But in this case $\left\{b_{\alpha}: \alpha \in A\right\}$ can be embedded in a dual ideal

$$
T=\left\{b: b \supseteq \bigcap_{i=1}^{n} b_{\alpha_{i}} \text { for some finite subset }\left\{\alpha_{i}\right\} \text { of } A\right\} .
$$


Also clearly $b \notin T$ since $\left\{\mathscr{M}\left(b_{\alpha}\right), h(b)\right\}$ have the FIP. So by the usual argument with Zorn's lemma it follows that there is a prime ideal $P$ with $b \in P$ and $P \cap T=\square$, $P \in \operatorname{Spec} B$ (Th. 6 of [6]). Now suppose $M$ is a minimal prime ideal such that $b \in M$ i.e. $M \in h(b)$. Then by (1), there is some $\alpha_{0} \in A$ such that $M \notin \mathscr{M}\left(b_{\alpha_{0}}\right)$. But this is just $b_{\alpha_{0}} \in M$. So $M \cap T \neq \square$ and $M \cap P^{c} \neq \square$. So equivalently if $M$ is a minimal prime ideal with $M \subseteq P$ then $b \notin M$.

Suppose now that the condition holds. To show that no complement of an element not in $Z(B)$ can be added to $B$.

Since $B$ is a disjunctive lattice, if $b_{1} \nsubseteq b_{2}$ then there is a minimal prime ideal $M$ with $b_{2} \in M$ and $b_{1} \notin M$. [Proof: $b_{1} \ddagger b_{2}$ implies that there exists a $c \in B$ such that $\square \neq c \subseteq b_{1}, c \cap b_{2}=\square$; hence the principal ideals $(c),\left(b_{2}\right)$ are disjoint. By Th. 8 of [6], there is a prime ideal $P$ such that $P \supseteq\left(b_{2}\right),(c) \nsubseteq P$. Then $b_{1} \in P$ would imply $c \in P$. Contradiction. A minimal prime ideal inside $P$ may then be selected.] So suppose that $P$ is the prime ideal corresponding to $b \notin Z(B)$. Index the elements of $P^{c}$ i.e. set

$$
P^{c}=\left\{b_{\alpha}: \alpha \in A\right\} \text { or }\left\{\mathscr{M}\left(b_{\alpha}\right): a \in A\right\} .
$$

Since $P^{c}$ is a prime dual ideal, clearly $\left\{\mathscr{H}\left(b_{\alpha}\right): \alpha \in A\right\}$ has the FIP, since dual ideals are closed under finite meets.

Also since $b \in P$ it follows that $b_{\alpha} \neq b$ for all $\alpha$. So for each $\alpha$ there is a minimal prime ideal $M_{\alpha}$ with $b \in M_{\alpha}, b_{\alpha} \notin M_{\alpha}$. But this means just that $M_{\alpha} \in h(b)$, $M_{\alpha} \in \mathscr{M}\left(b_{\alpha}\right)$.

A finite intersection of elements from $\left\{\mathscr{H}\left(b_{\alpha}\right), h(b)\right\}$ reduces to either $\mathscr{M}\left(b_{\alpha_{0}}\right) \cap h(b)$ or $\mathscr{M}\left(b_{\alpha_{0}}\right)$, because $\left\{\mathscr{M}\left(b_{\alpha}\right)\right\}$ is a dual ideal. We have just shown that $M_{\alpha_{0}} \in \mathscr{M}\left(b_{\alpha_{0}}\right) \cap h(b)$; consequently $\left\{\mathscr{M}\left(b_{\alpha}\right), h(b)\right\}$ has the FIP.

So $\left\{h(b), \mathscr{M}\left(b_{\alpha}\right)\right\}$ is the required FIP family which proves that $h(b)$ is not compact, using the condition assumed on $P$.

I would like to express my gratitude to Dr. T. P. Speed for his help and encouragement in this work, and also to the referee for his many valuable suggestions.

\section{References}

[1] T. P. Speed, Spaces of Ideals of Distributive Lattices II Minimal Prime Ideals, to appear.

[2] K. D. Magill Jr \& J. A. Glasenapp, '0-dimensional Compactifications and Boolean Rings', J. Aust. Math. Soc. 8 (1968), 755-765.

[3] A. Nerode, 'Some Stone Spaces and Recursion theory', Duke Math. J. 26 (1959), 397-406.

[4] Ph. Dwinger, Introduction to Boolean Algebras (Physica-Verlag 1961).

[5] J. Kist, 'Minimal Prime Ideals in Commutative Semigroups', Proc. London Math. Soc. (3) 13 (1963), 31-50.

[6] M. H. Stone, 'Topological Representations of Distributive Lattices and Brouwerian Logics', Casopic Pro Pestorani Matematiky a Fysiky 67 (1937), 1-25.

Monash University

Clayton, Victoria

Australia 